\title{
The challenge of stereo PIV measurements in the tip gap of a transonic compressor rotor with casing treatment
}

\author{
Melanie Voges ${ }^{1}$, Chris Willert ${ }^{1}$, Reinhard Mönig ${ }^{1}$, Martin W. Müller², \\ Heinz-Peter Schiffer ${ }^{2}$
}

\author{
1: Institute of Propulsion Technology, German Aerospace Center (DLR), Cologne, Germany, melanie.voges@dlr.de \\ 2: Dept. of Gasturbines and Aerospace Propulsion, Technische Universität Darmstadt, Germany, \\ mueller@glr.tu-darmstadt.de
}

\begin{abstract}
This contribution is aimed at summarizing the effort taken to apply stereoscopic PIV (SPIV) measurements in the tip clearance of a transonic compressor rotor equipped with a casing treatment. A light sheet probe was placed downstream of the stator and aligned to pass the light sheet through a stator passage into the blade tip clearance of the rotor. A setup with three cameras has been used in order to record the entire $2 \mathrm{C}$ velocity field and the smaller area of $3 \mathrm{C}$ field of view at the same time instance for comparison with earlier 2C PIV results. A homogeneous seeding distribution was achieved by means of a smoke generator, working with evaporated and subsequently re-condensed mineral oil. The main emphasis of the SPIV measurement was to establish a data set with high spatial resolution close to the compressor casing, where the aerodynamic effects of a CT are known to be strong. Additionally the SPIV data was intended to be used for comparison with numerical results and related code validation, as detailed laser-based flow field investigations in the vicinity of a casing treatment in a transonic compressor environment are barely reported in the literature.

The paper will discuss some major aspects of the utilized PIV data processing and point out a variety of frequently underestimated error sources that influence the overall quality of the recovered data in spite of the fact that the individual PIV recordings seemed to be of very good quality. Thus the authors will not focus on the PIV results and related interpretation of the flow field, but on the optimization and procedures applied during setup of the experiment and data processing respectively.
\end{abstract}

\section{Introduction}

A casing treatment (CT) is an effective measure to extend the stable operating range of high pressure compressor stages needed for today's turbomachinery applications, such as stationary gas turbines or aircraft engines (see Greitzer et al. 1979). While CT result in increased stage efficiency and/or extended stall margin the underlying aerodynamics are still not fully understood. A large number of numerical simulations can be found in the literature (e.g. Hathaway 2002, Zhu and Chu 2005, Emmrich et al. 2007) discussing compressor performance as well as aerodynamic effects. In order to validate the numerical models applied for CFD, the demands on accuracy and spatial resolution for non-intrusive laser-based flow diagnostics are steadily increasing to match the continuous improvement of numerical simulation tools. Especially the particle image velocimetry (PIV) technique has reached a high level of maturity and good accuracy during the recent years (Westerweel 2000, Wereley and Meinhart 2001) and is well-suited for turbomachinery applications (Raffel et al. 2007, Wernet 2000, Wernet et al. 2005, Woisetschläger et al. 2003). As PIV is capable of providing immediate topological insight to the flow structures as well as providing statistical information based on a sufficiently high number of instantaneous measurements, this technique today is frequently chosen for comparison with numerical simulations and related code validation.

In the present investigation PIV was applied to obtain detailed measurements in a transonic compressor rotor close to the casing, where the aerodynamic effects of a CT are known to be strong. The compressor stage, shown in Fig. 1, operated at Technische Universität Darmstadt was equipped with a casing treatment based on the geometry investigated by Zhu and Chu (2005). In contrary to 
Zhu and Chu (2005) the CT cavities have been radially aligned to improve optical access through the CT slots. Previous 2C PIV investigations revealed slight deviations of the flow field compared to numerical results, while the general agreement between CFD and PIV results was still very good (Schnell et al. 2008, Voges et al. 2008b). The differences are believed to originate from a strong radial velocity component in the tip region of the rotor blades close to the CT, with considerable motion across the interface plane between cavity and main flow. Due to the 2C PIV imaging geometry this motion could not be measured and motivated the use of stereoscopic PIV (SPIV) in a subsequent measurement campaign which is the main subject of the present paper.
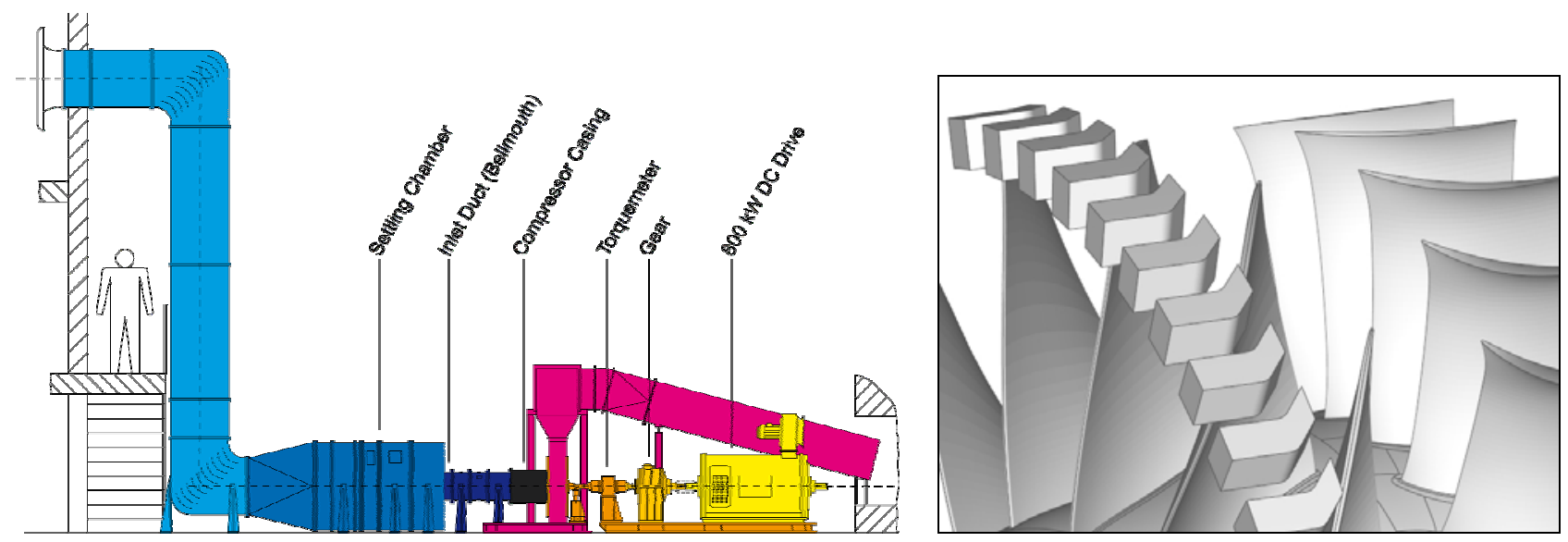

Fig. 1: left: Scheme of the transonic compressor facility at Technische Universität Darmstadt; right: transonic compressor stage with casing treatment, consisting of a rotor (titanium blisk with 16 blades), followed by a stator (29 CFRP vanes); aerodynamic design point (ADP) of the stage at a standard day-corrected rotational speed of 20,000 rpm

\section{Transonic Compressor Facility and Stereo PIV Setup}

The single-stage transonic axial compressor was equipped with a casing treatment (CT), consisting of 3.5 axial slots per rotor pitch in order to investigate the numerically predicted extension of the stall margin characteristics. Contrary to most other CT geometries (e.g. Yang et al.2003), the CT was specifically designed for an optimized optical access in the immediate vicinity of the CT, rather than giving maximum benefit in terms of stall margin extension. The nearly rectangular geometry of the CT cavities, as shown in the Fig. 1 (right), allowed one dividing bridge between two slots to be made of quartz glass with curvatures matching the inner contour of the casing. Thus the flow phenomena could be observed with essentially no disturbance caused by the optical access. The rotor was covered with a thin layer of black paint in order to reduce possible laser reflections.

Following the experience made during the 2C PIV measurements described in Voges et al. 2008a, the setup has been extended by using a set of three cameras, with one camera viewing the light sheet orthogonally and two cameras in a symmetrical arrangement for SPIV measurements. The recessment of the optical access into the casing and space constraints on the facility limited the optical access to one single CT slot, as shown in Fig. 2 (right) and restricted the stereo arrangement of the observing camera system to relatively small viewing angles with $\alpha= \pm 24^{\circ}$ from the light sheet normal to keep the field of view to the maximum possible. Due to space constraints in the vicinity of the compressor rig one of the cameras observed the flow field with the help of a mirror, as depicted in the photograph of Fig. 2. The demand for high spatial resolution was accounted for using macro zoom lenses with $105 \mathrm{~mm}$ focal length for the two stereo cameras and $85 \mathrm{~mm}$ optics for the normal viewing direction. For flow observation thermo-electrically cooled, double-shutter CCD cameras (pco.1600, PCO AG) with a sensor spatial resolution of 1,600 x 1,200 px at a frame 
rate of $15 \mathrm{~Hz}$ were used. All three cameras were mounted on Scheimpflug adaptors to allow for optimized alignment of the camera optics with the laser light sheet plane, not only for the stereoscopic arrangement but also for the light sheet normal viewing direction. In the observation area of one single CT cavity the access window is flat with parallel glass surfaces.
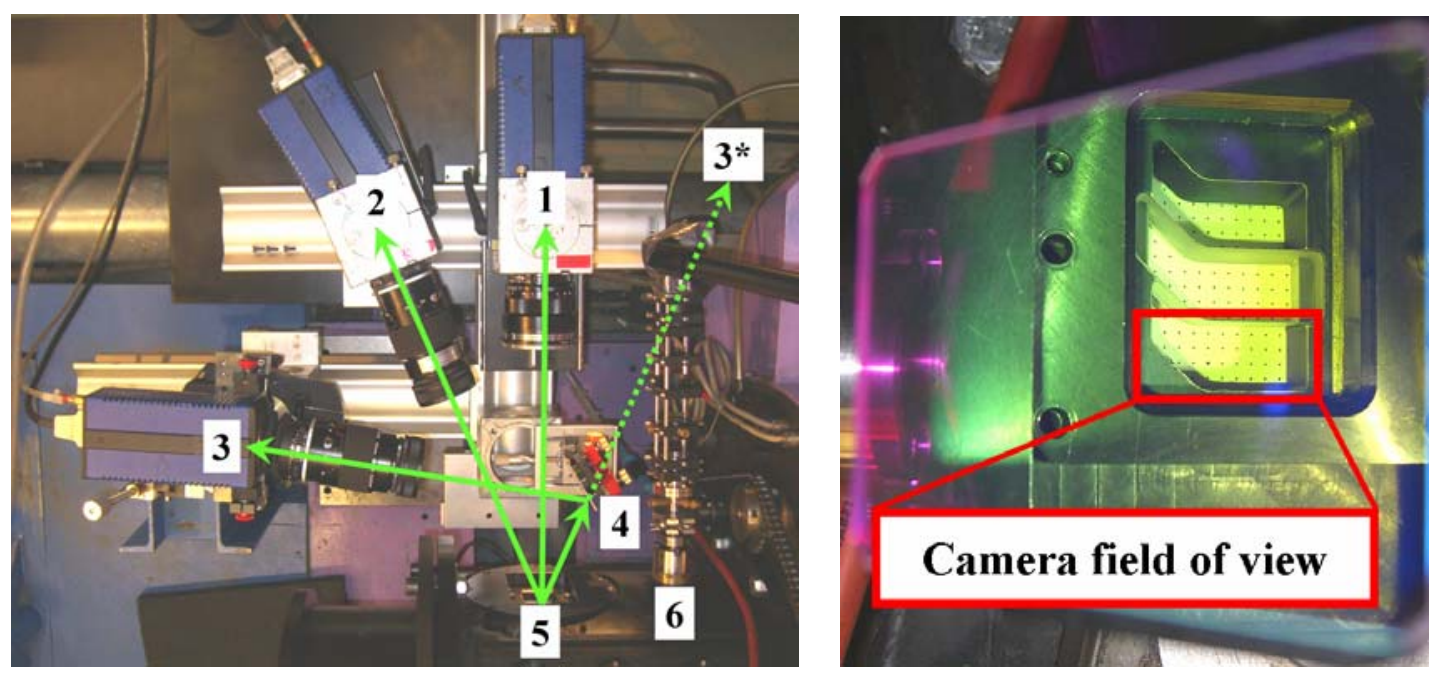

Fig. 2: left: Combined $2 \mathrm{C}$ and $3 \mathrm{C}$ PIV setup using a set of three cameras: a normal viewing camera (1) and two offaxis cameras (2, 3). Mirror (4) is used for camera (3). Optical access integrated into a casing plug (5). Periscope probe (6); right: mirror view of camera (3) and final field of view

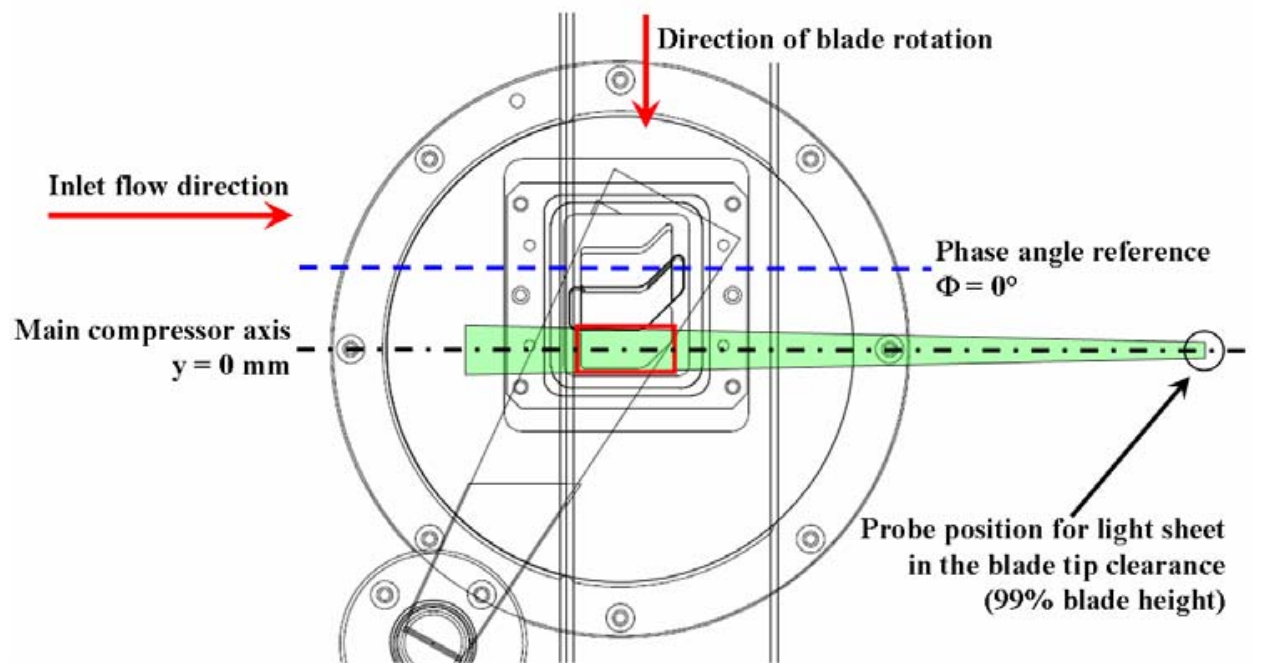

Fig. 3: Window support and light sheet probe position in the compressor casing related to cameras field of view (red rectangle); black line (dot-dashed): axial reference $y=0 \mathrm{~mm}$ for radial light sheet position and light sheet normal camera viewing direction; blue line (dashed): phase angle reference $\Phi=0^{\circ}$

A homogeneous and stable tracer particle distribution was achieved with the help of a smoke generator relying on evaporation and re-condensation of special mineral oil. The generator was placed in the inlet settling chamber upstream of the compressor stage. The particle size distribution was quoted by the manufacturer to be in the order of $300-800 \mathrm{~nm}$. In comparison to seeding provided by atomizer based systems the particle response time in the strong gradients (shock) of the transonic compressor flow field could be reduced by a factor of $2-4$ (Voges et al. 2007).

For illumination of the tracer particles in the light sheet a commonly available, dual cavity, frequency doubled Nd:YAG PIV laser with $120 \mathrm{~mJ}$ pulse power at $532 \mathrm{~nm}$ wavelength was operated at $15 \mathrm{~Hz}$ repetition rate. An articulated mirror arm was installed in order to optimize the alignment between laser heads and optics. The rigid coupling between light sheet optics, light sheet 
probe and casing made the entire set up very tolerant to machine vibrations and movement. Of course the $15 \mathrm{~Hz}$ repetition rate of the laser system was not enough for directly resolving typical flow structures at blade passing frequencies (BPF) in a range of $3.4-5.4 \mathrm{kHz}$. Phase-constant PIV measurements were obtained by feeding a rotor blade trigger signal into a phase-shifting trigger unit. Each rotor passage was divided into 9 discrete phase angles: 8 equidistant phase steps plus one step with the rotor leading edge placed in the center of the CT slot. Given a phase angle of $360^{\circ}$ corresponding to one full rotor revolution, the trigger for phase-constant PIV measurements could be adjusted with a precision of $0.2^{\circ}$, corresponding to a phase uncertainty of $6.3 \%$ related to the phase increment of 2.8 . A number of 970 images (limited by the internal memory of the PIV cameras) were recorded for each phase angle and operating condition, respectively. For better comparison of the data obtained the rotor reference position $\Phi=0^{\circ}$ has been kept from earlier investigations, as indicated in Fig. 3.

An adjustable periscope light sheet probe with integrated optics was used to guide the laser beam through the casing and to support the alignment of the light sheet in the tip gap of the rotor. The probe support was built in a standard casing plug downstream of the stator. The stator had to be traversed and fixed in a suitable position for passing the laser beam through the vane passage. Thus any clocking investigation related to the CT were omitted and performed subsequently to the SPIV measurements. As the tip cap of the light sheet probe contained the laser beam deflecting mirror, it was necessary that the cap entered the passage flow field. To reduce any possible upstream disturbance the outer diameter was kept to minimum of $12 \mathrm{~mm}$, resulting in very small optical components with diameters below $6 \mathrm{~mm}$. That required a precise alignment of the laser beam path through the probe to avoid streaking and associated laser flare from the inner surface of the probe. This was achieved with a collimating lens pair for beam diameter adjustment along with two cylindrical lenses which formed the light sheet with a waist thickness of $1 \mathrm{~mm}$ and a divergence angle of about $7^{\circ}$ that covered the width of a single CT slot. A schematic of the light sheet optics is depicted in Fig. 4 together with a cross sectional drawing of the periscope probe. The probe was purged with clean, dry and oil-free air in order to prevent possible deposits of aerosol seeding or dust on and subsequent damage to the exposed optical surfaces.

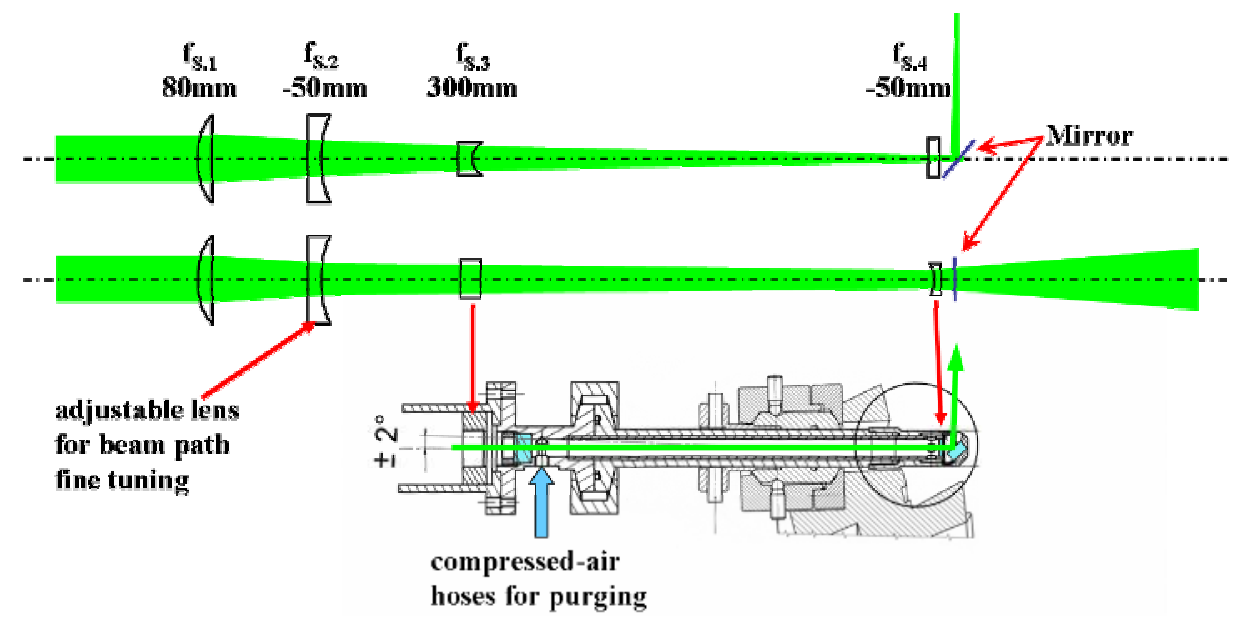

Fig. 4: Schematic of the periscope probe and the light sheet optics (not drawn to scale), consisting of a collimating lens pair ( $f_{\mathrm{S} .1}$ and $\left.\mathrm{f}_{\mathrm{S} .2}\right)$ and a set of two cylindrical lenses with orthogonal focal planes $\left(\mathrm{f}_{\mathrm{S} .3}\right.$ and $\left.\mathrm{f}_{\mathrm{S} .4}\right)$

\section{Alignment and Calibration}

For camera calibration a precise dot grid with $2.0 \times 2.0 \mathrm{~mm}$ square spacing was placed in the tip clearance of the rotor. Limited access to the rotor passage prevented the use of a translated or multilevel calibration target that is frequently used in stereo PIV calibration (Soloff \& Adrian, 
1997). Thus camera calibration had to be performed using only a single calibration plane (Willert, 2006). The grid reference point was positioned in the center of the slot and above the trace of the rotor leading edge as this position was visible for all three camera viewing directions. Due to the high magnification and the narrow geometry of the CT cavity only a low number of grid nodes were visible in the stereoscopic camera recordings. Therefore utmost care was taken during the image mapping procedure for best-possible grid detection and dewarping. Additionally a polynomial, $2^{\text {nd }}$ order disparity correction scheme was applied to the particle image recordings in order to correct for slight misalignment between light sheet plane and camera focal planes, which can not completely be avoided in non-laboratory, "real-life" experiments.

In general this setup was very susceptible to any kind of misalignment, especially in terms of laser beam alignment and beam overlap. The latter was accounted for using a CCD-based beam profiler to adjust the beam overlap and optimize the matching of the intensity profiles of both laser beams. As one of the two beams had an elliptical intensity profile, the articulated mirror arm was used to rotate the laser beam in order to find the best possible, homogeneous intensity distribution for the final light sheet. Due to manufacturing tolerances the beam deflection at the probe tip mirror was limited to $88^{\circ}$ rather than the required $90^{\circ}$. As the mirror was permanently glued into the tip cap of the periscope probe using temperature resistant, epoxy bonding material, the deflection angle could not easily be corrected for. To overcome this problem the aperture of the laser beam exit at the tip of the probe was milled (with mirror installed) in order to increase the exit area. With the help of a \pm 2 degree pivoting mechanism of the probe support and the adjustable lens in the beam path at the probe inlet it was possible to adjust the light sheet through the tip clearance of the rotor. As the width of the tip clearance was in the order of the light sheet thickness, streaking of the laser sheet on the compressor casing could not be entirely avoided. Thus a weak reflection effect occurred and low-intensity side peaks appeared in the vicinity of the light sheet. This was accounted for by setting the lens apertures to f\# numbers of 4 and 5.6 which limited the depth of field to the main intensity of the light sheet $\left(1 / \mathrm{e}^{2}\right.$ criterion).

\section{Discussion of Error Sources}

The PIV recordings showed high quality in terms of contrast and particle image homogeneity. Image areas without velocity information (e.g. window brace or parts of the CT ring) were masked. The sample images given in Fig. 5 show a dense and homogeneous particle distribution and only slight variation of the overall intensity due to forward and backward scattering observation. Areas with window contamination, close to the center of all three recordings, appear light-grey. Particle images in that region seem to be blurred, out of focus or smeared out in a preferred direction due to oil droplets deposited on the glass surface during compressor operation. Single pass cross correlation processing of a subset of 50 images revealed that the signal disturbance due to this astigmatism was small and therefore did not contribute to the calculated particle displacements. This can be explained by the fact that the light sheet is close to the distorting surface $(\sim 10 \mathrm{~mm})$ in comparison to the camera receiving lens $(\sim 300 \mathrm{~mm})$ such that angular deflections are minimal. For camera 3, observing the flow field in a backward scattering arrangement, a strong laser reflection appeared where the light sheet illuminates the bottom edge of the CT cavity. This is visible as a dark area on the left part of the image recorded by camera 3 (Fig. 5). Stereo reconstruction involving displacement data from camera 3 introduced a dominant out-of-plane velocity component into the CT slot (Fig. 6), that did not seem physically plausible. In this region the residuals of the 3$\mathrm{C}$ reconstruction are in the order of $15-20 \mathrm{px}$, which is a clear indication of questionable stereo reconstruction results. The absolute velocity values even exceeded the magnitude of the radial velocity component (in the order of the free rotor inlet velocity) predicted by former numerical simulations. Even the subtraction of a background image did not improve the evaluation results. Other portions of the imaged areas exhibit 3C residuals below 2.0 px (see Fig. 9, bottom right part 
of the displayed PIV data), which is acceptable for the given measurement conditions.
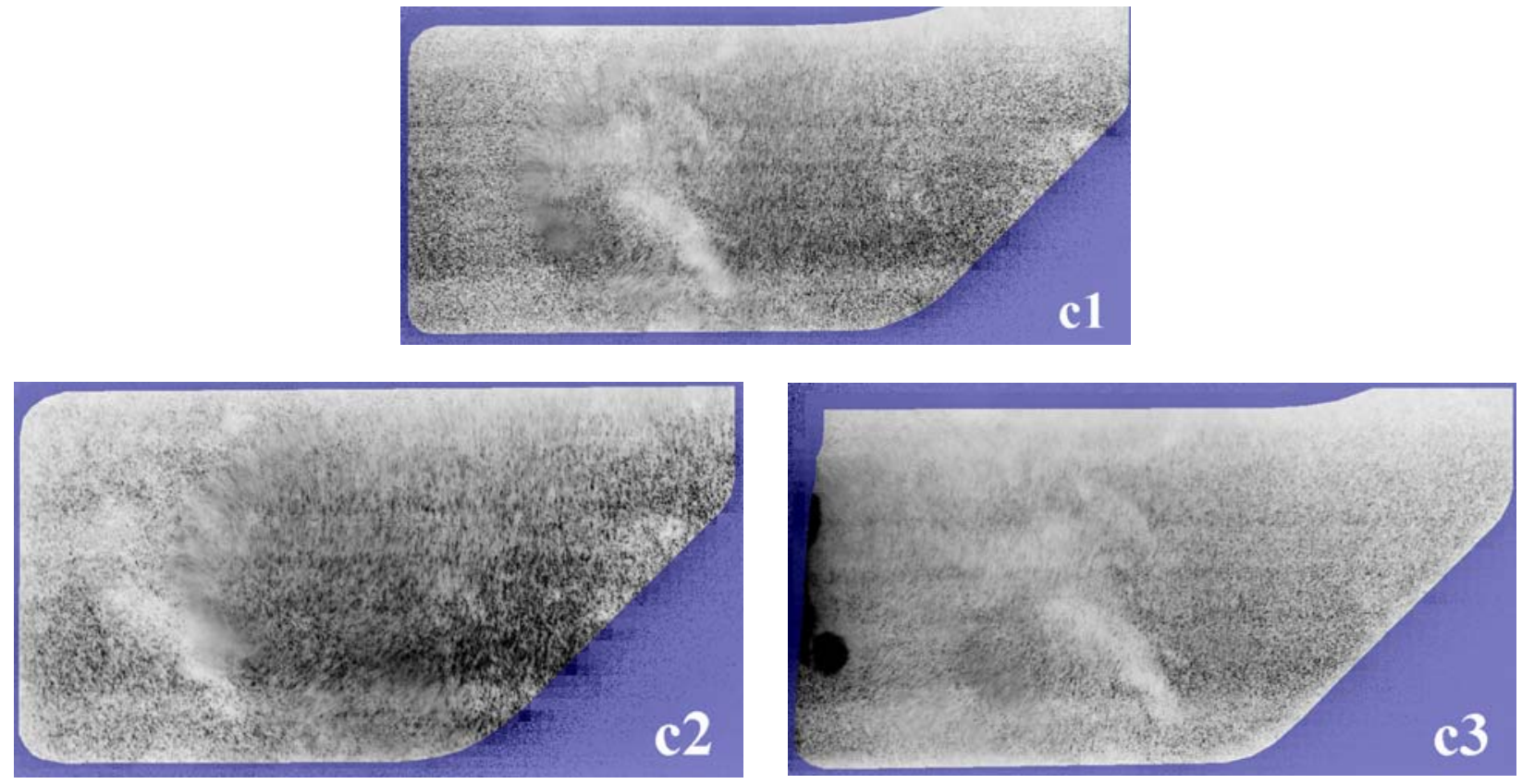

Fig. 5: Raw particle images after dewarping (inverted grey scale for better visibility, dark areas are brightest); masked image areas are displayed in blue color; c1: light sheet normal viewing direction; c2: forward scattering stereo camera view; c3: backward scattering stereo camera view

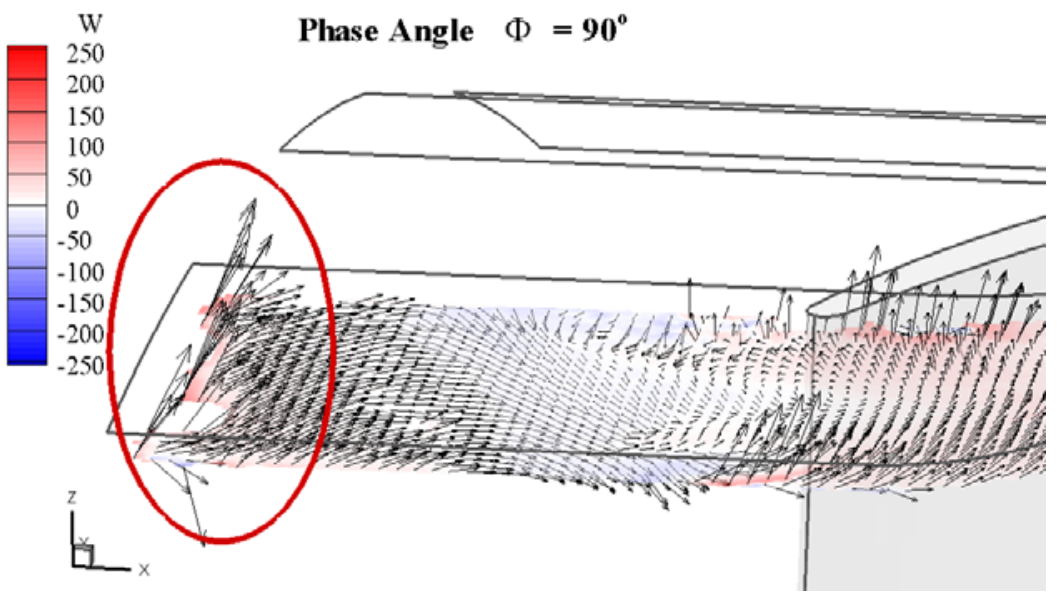

Fig. 6: Over-estimated, erroneous out-of-plane component in the $3 \mathrm{C}$ vector field, identified by $3 \mathrm{C}$ residuals $>15 \mathrm{px}$; areas with strongest laser reflections have already been blanked (number of shown vectors reduced by a factor of 4 for better visibility)

In order to identify the error source more precisely, the stereo vector reconstruction has been performed manually following the least squares approach by Raffel et al. (2007) according to Fig. 7. Based on some example PIV data sets the displacement values have been varied using simple Matlab $\subset$ matrix and vector operation tools and compared to the observed behavior of the actual PIV data. This procedure revealed that the high out-of-plane velocities are directly related to a dominant, erroneous X-component of the particle shifts introduced by the laser reflections. A crosscheck with the undisturbed 2C particle displacements of camera 1 showed the expected physical behavior of the flow field. Originally it was planned to evaluate the SPIV data in three steps, combining first the data of cameras 2 and 3, then cameras 1 and 2 and finally cameras 1 and 3. All three stereo data sets should subsequently be combined, in order to provide 3C results even in the 
regions not visible in the "ideal", symmetric stereo-combination of cameras 2 and 3, as well as to reduce the measurement uncertainty in the common image area of all three views. Given the findings of the manual stereo reconstruction on the one hand and the increased uncertainty combining camera 1 with camera 2 or 3 respectively, a reasonable and reliable 3C velocity field calculation can only be performed based on the stereo imaging results of cameras 2 and 3.

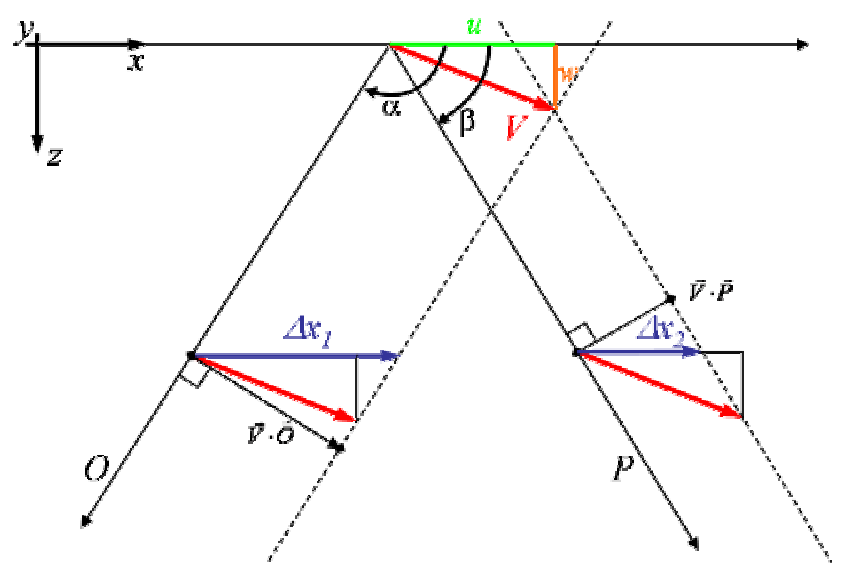

$$
\begin{aligned}
& {\left[\begin{array}{l}
\Delta x_{1} \\
\Delta y_{1} \\
\Delta x_{2} \\
\Delta y_{2}
\end{array}\right]=\left[\begin{array}{rrr}
1 & 0 & -\frac{O_{x}}{O_{z}} \\
0 & 1 & -\frac{O_{y}}{O_{z}} \\
1 & 0 & -\frac{P_{x}}{P_{z}} \\
0 & 1 & -\frac{P_{y}}{P_{z}}
\end{array}\right] \cdot\left[\begin{array}{c}
u \\
v \\
w
\end{array}\right]} \\
& \Delta \vec{X}=\mathbf{A} \cdot \vec{V} \\
& \vec{V}=\left(\mathbf{A}^{T} \mathbf{A}\right)^{-1} \mathbf{A}^{T} \Delta \vec{X}
\end{aligned}
$$

Fig. 7: left: Stereo reconstruction scheme; right: related equations after image mapping and least squares approach (according to Raffel et al. 2007)
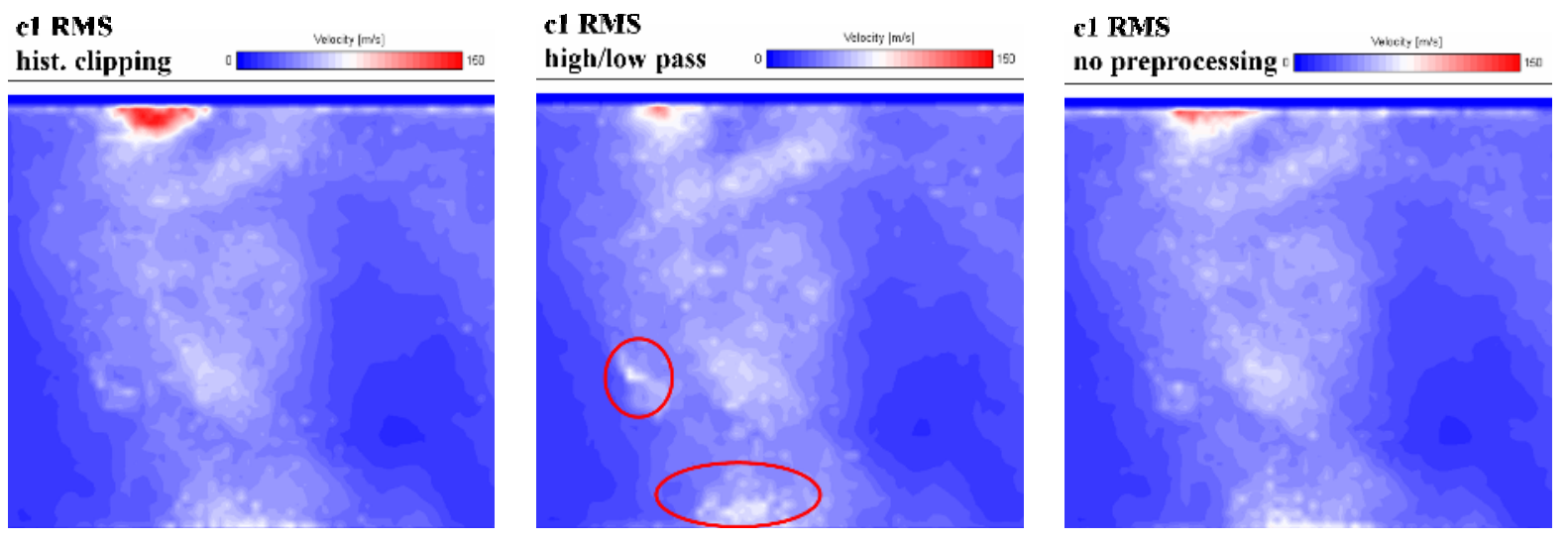

Fig. 8: RMS values of the absolute velocity based on an average of 50 images left: preprocessing with histogram clipping introduced over-estimated RMS values in the top-right part of the image; middle: combination of high and low pass filtering induced artifacts while peak values of RMS distribution are under-estimated; right: RMS values of the original data set without any preprocessing applied

The calculation of the velocity fields was performed applying a cross-correlation algorithm including grid refinement, starting with an interrogation window size of $64 \times 64 \mathrm{px}$ and a final window size of $24 \times 24$ px using $50 \%$ overlap. Sub-pixel accuracy was accounted for applying iterative image-shifting routines with Whitaker reconstruction for sub-pixel peak position estimation ("sinc"-kernel interpolation, Roesgen 2003). Outlier detection was based on normalized median filtering (Westerweel and Scarano 2005). Image preprocessing was not applied, since the most common types of image filters (high/low pass, histogram clipping, see Shavit et al. 2007) were found to increase background noise effects and additionally introduced peak locking or other artifacts that were visible in the RMS values (Fig. 8).

Fig. 9 shows a comparison of the present 3C measurements with respect to earlier 2C measurements, which had a spatial resolution of $34 \mathrm{px} / \mathrm{mm}, 32 \mathrm{x} 32 \mathrm{px}$ window size and $50 \%$ overlap. The present SPIV exhibit a spatial resolution of $44 \mathrm{px} / \mathrm{mm}$ yielding a vector spacing of $0.3 \mathrm{~mm}$ based on an interrogation window size of $24 \mathrm{x} 24 \mathrm{px}$ at $50 \%$ overlap. The resolution could be 
even increased to $0.2 \mathrm{~mm}$ vector spacing, applying an ensemble averaging approach including multi pass algorithm with $16 \times 16 \mathrm{px}$ window size at 50\% overlap (Willert, 2008). Since the ensemble averaging algorithm does not provide any statistical information such as RMS values or vector count, the single pair evaluation algorithm was chosen according to the desired aspects of flow analysis and interpretation of the SPIV results. In the present investigation knowledge of the RMS distribution of the velocity was needed in order to give a rough estimate of turbulent flow characteristics.

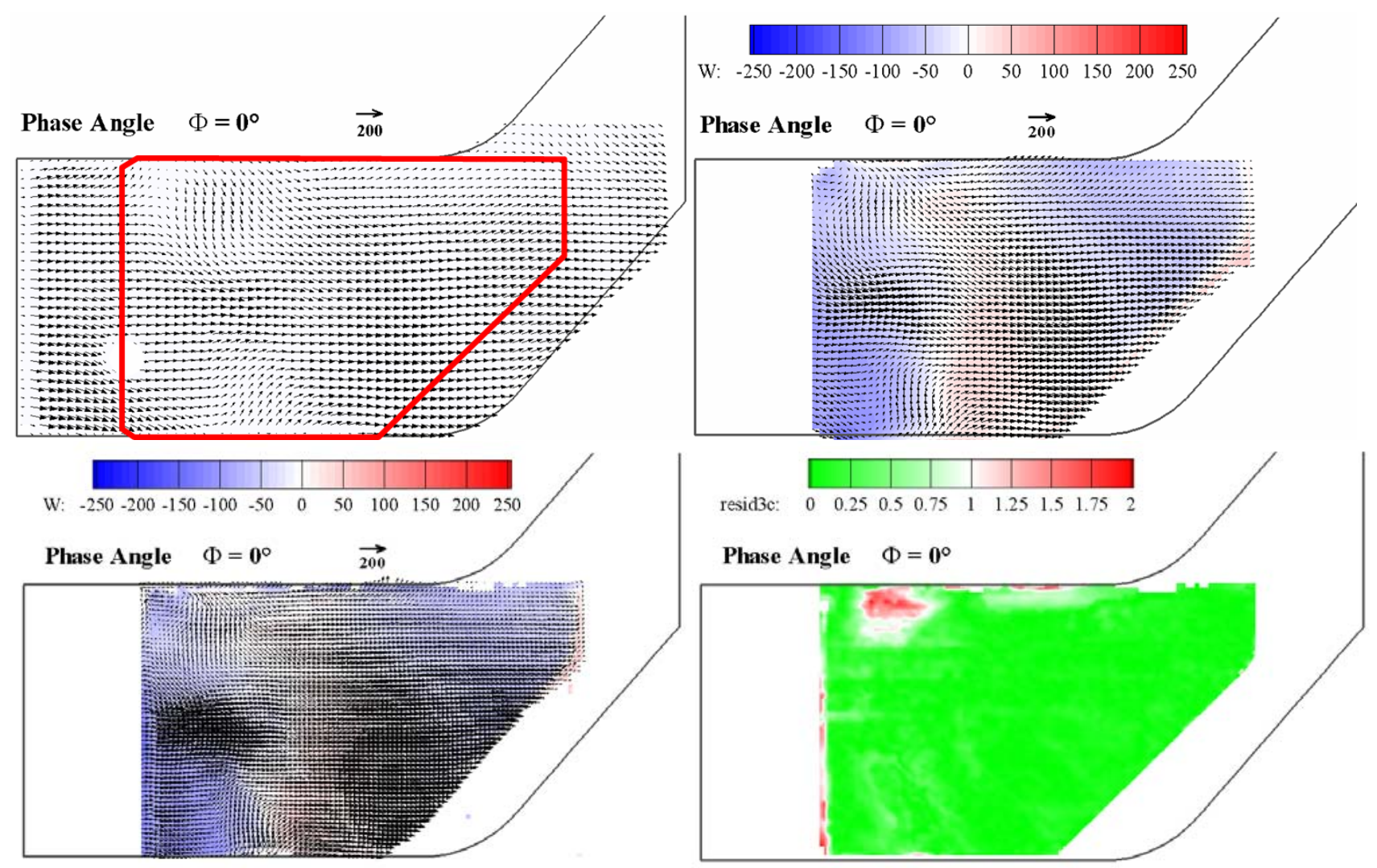

Fig. 9: top left: Former 2C PIV results with a grid distance of $0.5 \mathrm{~mm}$, red polygon indicating the field of view for 3C results; top right: improved resolution for SPIV results with a grid distance of $0.3 \mathrm{~mm}$; bottom left: ensemble average of the SPIV data with a final vector spacing of $0.2 \mathrm{~mm}$; bottom right: vector residuals of the stereo reconstruction based on the ensemble averaged SPIV results

The measurement error can be estimated assuming a $0.1 \mathrm{px}$ measurement uncertainty (Westerweel, 2000). According to Prasad (2000) both camera views contribute symmetrically to the measured values, thus the in-plane measurement error is reduced by a factor of $1 / \sqrt{2}$, assuming identical standard deviation for both, $\mathrm{x}$ - and $\mathrm{y}$-component of the measured displacements. For the out-of-plane particle shifts, the relative measurement error scales with $1 / \arctan \alpha$, where $\alpha$ describes the angle between the light sheet normal and the camera viewing direction (symmetrical camera arrangement in this special case). Given particle displacements of $12.5 \mathrm{px}$ (in-plane) and $\pm 8 \mathrm{px}$ (out-of-plane) at a laser pulse separation of $1-1.5 \mu$ s and a magnification of $44 \mathrm{px} / \mathrm{mm}$, this yields measurement errors as follows

$$
\begin{aligned}
\sigma_{\Delta x} & =\sigma_{\Delta y}=1.7 \frac{\mathrm{m}}{\mathrm{s}} \\
\sigma_{\Delta z} & =5.5 \frac{\mathrm{m}}{\mathrm{s}}
\end{aligned}
$$

Taking into account the non-ideal stereo viewing arrangement of the cameras and the strong 
laser reflections present in the recordings of camera 3, the real out-of-plane measurement uncertainty is much higher, yielding $27.5 \%$ for image areas with vector residuals of $2 \mathrm{px}$. Under ideal stereoscopic imaging conditions (see Prasad 2000: 45 deg viewing direction related to the light sheet normal) this high measurement uncertainty of about $50 \mathrm{~m} / \mathrm{s}$ in the absolute domain could have been reduced to acceptable values. Unfortunately, due to space constraints in the close environment of the compressor facility, larger viewing angles could not be realized, while laser reflections could be removed using only the common image area of camera 2 and 3 for stereo reconstruction.

To the authors' knowledge the herein presented combined PIV and SPIV measurements in the vicinity of a casing treatment in a transonic compressor stage, have not yet been reported in the literature. The obtained results form a valuable data base for comparison with numerical results and related code validation in turbomachinery related research.

\section{Summarizing Comments}

A combined setup allowing for standard 2C PIV and stereoscopic PIV measurements at the same time instance has been applied to a transonic compressor rig with casing treatment, involving three CCD cameras and a periscope light sheet probe. The setup was designed to minimize the influence of the applied measurement equipment on the flow field under observation. The flow investigations in the tip clearance of the rotor were aimed at providing detailed $3 \mathrm{C}$ flow field information at high spatial resolution in order to improve the understanding of the interaction between rotor passage flow and the casing treatment. Although the initial quality of the individual PIV recordings seemed to be of high quality in terms of homogeneous particle distribution, scattered light intensity and contrast, some initially underestimated error sources significantly affected the quality of the recovered three-component velocity data. Laser flare on the facility casing affected the camera observing the flow in a backward scattering arrangement, which introduced an erroneous in-plane particle displacement. Hence the stereo reconstruction calculated very high out-of-plane velocity components that were not physically plausible. Therefore the outer image regions of the CT slot could not be used for calculation of the $3 \mathrm{C}$ vector field. Due to limited space in the close environment of the compressor stage the camera viewing angles were restricted to small values ( $\pm 24^{\circ}$ from the light sheet normal), resulting in increased vector residuals of up to $2 \mathrm{px}$ after stereo reconstruction. This dramatically increased the relative measurement uncertainty of the out-of-plane component from initially $3.0 \%$ to $27.5 \%$, although the $2 \mathrm{C}$ velocity results obtained simultaneously to the $3 \mathrm{C}$ data show a very low measurement error of only $0.6 \%$.

In a next step the 2C and SPIV results will be further analyzed and prepared for comparison with CFD results of the same compressor geometry. Subsequent to a quantification of the remaining error sources of both, measurement and numerical simulation, the combined results of PIV and CFD are believed to provide a deep insight as well as better understanding to the complex flow phenomena present in transonic compressor stages.

\section{Acknowledgements}

The authors gratefully acknowledge the good cooperation with MTU Aero Engines GmbH, namely Dr. C. Zscherp, who designed the casing treatment during a former research program in the framework of AG TURBO COOREFF-T (reference number $0327713 \mathrm{E}$ ). Based on the encouraging outcomes of this project the PIV investigations have been continued. 


\section{References}

Emmrich, R., Hoehnen, H., Niehus, R. (2007): Time Resolved Investigations of an Axial Compressor with Casing Treatment, ASME Turbo Expo 2007 Power for Land, Sea and Air, paper GT2007-27582, May 14-17, Montreal/ Canada

Greitzer, E.M., Nikkanen, J.P., Haddad, D.E., Mazzaway, R.S., Joslyn, H.D. (1979): A Fundamental Criterion for the Application of Rotor Casing Treatment. ASME Journal of Fluids Engineering 101: 237-243

Hathaway, M.D. (2002): Self-Recirculating Casing-Treatment Concept for Enhanced Compressor Performance, ASME Turbo Expo 2002 Power for Land, Sea and Air, paper GT2002-30368, June 03-06, Amsterdam/ NL

Prasad, A.K. (2000): Stereoscopic Particle Image Velocimetry. Exp. Fluids 29:103-116

Raffel, M., Willert, C., Wereley, S., Kompenhans, J. (2007): Particle Image Velocimetry, a practical guide (Second Edition), Springer Berlin Heidelberg, ISBN 978-3-540-72307-3

Roesgen, T. (2003): Optimal Subpixel Interpolation in Particle Image Velocimetry. Exp. Fluids 35: 252-256

Schnell, R., Voges, M, Mönig, R. Mueller, M.W. and Zscherp, C. (2008): Investigation of Blade Tip Interaction with Casing Treatment in a Transonic Compressor - Part 2: Numerical Results, ASME Turbo Expo 2008 Power for Land, Sea and Air, paper GT2008-50212, June 09-13, Berlin/ Germany

Shavit, U., Lowe, R.J., Steinbuck, J.V. (2007): Intensity Capping: a simple method to improve cross-correlation PIV results. Exp. Fluids 42:225-240

Soloff, S.M., Adrian, R.J. (1997): Distortion compensation for generalized stereoscopic particle image velocimetry, Meas Sci Technol 8:1441

Voges, M., Beversdorff, M., Willert, C., Krain, H. (2007): Application of Particle Image Velocimetry to a Transonic Centrifugal Compressor, Exp. Fluids 43:371-384

Voges, M., Willert, C., Mönig, R. Mueller, M.W. and Zscherp, C. (2008a): PIV Application for Investigation of the Rotor Blade Tip Interaction with a Casing Treatment in a Transonic Compressor Stage. 14th Int. Symp. on Applications of Laser Techniques to Fluid Mechanics, July 07-10, Lisbon/ Portugal

Voges, M, Schnell, R., Willert, C., Mönig, R. Mueller, M.W. and Zscherp, C. (2008b): Investigation of Blade Tip Interaction with Casing Treatment in a Transonic Compressor - Part 1: Particle Image Velocimetry, ASME Turbo Expo 2008 Power for Land, Sea and Air, paper GT2008-50210, June 09-13, Berlin/ Germany

Wereley, S.T., Meinhart, C.D. (2001): Second-Order Accurate Particle Image Velocimetry. Exp. Fluids 31: 258-268

Wernet, M.P. (2000): Application of DPIV to Study Both Steady State and Transient Turbomachinery Flows, Optics \& Laser Technology 32: 497-525

Wernet M.P., van Zante, D., Strazisar, T.J., John, W.T., Prahst, P.S. (2005): Characterization of Tip Clearance Flow in an Axial Compressor Using 3-D Digital PIV, Exp. Fluids 39: 743-754

Westerweel, J. (2000): Theoretical Analysis of the Measurement Precision in Particle Image Velocimetry. Exp. Fluids 29: Suppl. S3-S12

Westerweel, J., Scarano, F. (2005): Universal Outlier Detection for PIV Data. Exp. Fluids 39: 10961100

Willert, C. (2006): Assessment of camera models for use in planar velocimetry calibration. Exp. Fluids 41: 135-143

Willert, C. (2008): Adaptive PIV processing based on ensemble correlation. 14th Int. Symp. on Applications of Laser Techniques to Fluid Mechanics, July 07-10, Lisbon/ Portugal

Woisetschläger, J., Mayrhofer, N., Hampel, B., Lang, H., Sanz, W. (2003): Laser-Optical 
Investigation of Turbine Wake Flow, Exp. Fluids 34: 371-378

Yang, H., Nürnberger, D., Nicke, E., Weber, A. (2003): Numerical Investigation of Casing Treatment Mechanisms with a Conservative Mixed-Cell Approach, ASME Turbo Expo 2003 Power for Land, Sea and Air, paper GT2003-38483, June 16-19, Atlanta/GA

Zhu, J. and Chu, W. (2005): The Effects of Bend Skewed Groove Casing Treatment on Performance and Flow Field Near the Endwall of an Axial Compressor, AIAA 2005-809, January 10-13, Reno/ Nevada 